\title{
Rhubarb alleviates acute lung injury by modulating gut microbiota dysbiosis in mice
}

\section{Tingyu Tang}

Zhejiang hospital

Fei Wang

Zhejiang hospital

Juan Liu

zhejiang hospital

Tian Zhao

zhejiang hospital

Wu Ye

Zhejiang Hospital

Zhijun Li ( Izj13575748493@sina.com )

Zhejiang Hospital https://orcid.org/0000-0002-1825-3781

\section{Research}

Keywords: Rhubarb, Acute lung injury, Gut microbiota, Th17/Treg, HDAC6

Posted Date: October 23rd, 2020

DOl: https://doi.org/10.21203/rs.3.rs-91447/v1

License: (c) (i) This work is licensed under a Creative Commons Attribution 4.0 International License. Read Full License

Version of Record: A version of this preprint was published at Current Microbiology on February 26th, 2022. See the published version at https://doi.org/10.1007/s00284-022-02811-x. 


\section{Abstract}

Purposes: Intestinal microbiota disorders can aggravate pulmonary inflammation during acute lung injury (ALI). Rhubarb, a Chinese herb, can regulated the gut microbiota. Therefore, this study was conducted to test the hypothesis that rhubarb alleviates gut microbiota dysbiosis and inflammation.

Methods: Feces were collected from patients with ALI to detect the gut microbiota using 16S rDNA sequencing. Subsequently, a mouse model of ALI was established using lipopolysaccharide to investigate changes in the gut microbiota, the periperal blood was attained for detecting the Th17/Treg cell ratio, and the effect of rhubarb treatment on the gut microbiota and Th17/Treg ratio were also evaluated.

Results: Rhubarb treatment significantly reduced the Firmicutes abundance and markedly increased the Bacteroidetes abundance in ALI patients. The rat models also showed a similar imbalance in the Firmicutes/ Bacteroidetes ratio. Rhubarb treatment alleviated the damaged lung tissue, accelerated Firmicutes proliferation, increased the HDAC6 in both the mice lung tissue and serum, and markedly reduced the Treg cells and increased the Th17 cells in the spleen tissue.

Conclusion: We determined that both patients with ALI and mouse models of ALI presented gut microbiota dysbiosis and Th17/Treg cell imbalances. Rhubarb promoted Firmicutes proliferation, increased the HDAC6 concentration, restored the Th17/Treg cell balance, and protected against ALI.

\section{Introduction}

The lungs are the most vulnerable target organ and are usually damaged early during sepsis and pulmonary injuries. Acute lung injury (ALI) and its more severe clinical manifestation, acute respiratory distress syndrome (ARDS), are common responses to various infectious and noninfectious etiologies, including severe sepsis, pneumonia, lung abscesses and severe acute pancreatitis, and can lead to uncontrollable inflammation with a cascade effect [1]. Progressive hypoxemia and respiratory distress syndrome are common clinical manifestations in patients with ALI. Despite the development of various therapeutic techniques, the mortality rate from ARDS can reach 70-90\% [2]. In 2005, the incidence of ALI increased to 306 per 100,000 person-years for people aged 75-84 years in the United States [3].

The human body contains numerous microflora, equaling 10 times the number of human cells. $\mathrm{A}$ "healthy gut microbiota" comprises a diverse range of intestinal microorganisms, which depend on host and environmental interactions [4]. A healthy microbiota protects against dysbiosis-related diseases, such as allergic sensitization, eczema, and asthma [5]. In patients with ALI/ARDS, the intestinal microbiota are thought to be disordered and aggravate inflammation in the lungs [6-8]. Increasing evidence indicates that a Th17/Treg cell imbalance is related to the development of several disorders, and patients with ALI exhibit increased Th17 cells [9]. A healthy gut microbiota and its metabolites contribute to regulating the Th17/Treg cell balance via epigenetic mechanisms [10]. Luo et al. showed that regulating the intestinal microbiota dysbiosis increased the short-chain fatty acid (SCFA) levels and restored the Th17/Treg cell 
balance [11]. Therefore, alterations in specific gut microorganisms and the effect of metabolites on the Th17/Treg cell imbalance and inflammation during LPS-induced ALI should be explored.

Rhubarb, a traditional herb with various pharmacological activities, palying anti-inflammatory effects, reduces intestinal permeability and bacterial translocation, and modulates gut microbiota dysbiosis [12]. In the gut, rhubarb supplementation improved intestinal ecosystem disorders and induced antimicrobial peptide expression [13]. Rhubarb also attenuated intestinal microbiota dysbiosis, relieved intestinal mucosal barrier damage, and inhibited intestinal inflammatory responses during acute pancreatitis [14]. Therefore, rhubarb regulated the gut microbiota and protected against ALI. Emodin, extracted from rhubarb, has shown anti-inflammatory properties for treating pancreatitis, atherosclerosis, asthma, and ALI [15-17]. Xiao et al. indicated that emodin relieved pulmonary edema and MCP-1 and E-selectin secretions and inhibited LPS-induced pulmonary damage [17]. Furthermore, emodin was shown to alter the gut microbiota structure, reduce the number of harmful bacteria, increase the number of beneficial bacteria, and ameliorate chronic kidney disease [18]. However, few published studies have explored the effect of rhubarb on the gut microbiota and inflammation during ALI development.

We hypothesized that rhubarb can alleviate intestinal microflora disorders and inflammation during ALI. To test this hypothesis, we evaluated gut microbiota dysbiosis in patients with ALI. We also used a mouse model of ALI induced by intratracheal administration of LPS to investigate changes in the intestinal microbiota and the Th17/Treg cell ratio. We also explored the effect of rhubarb on the gut microbiota and inflammation.

\section{Methods}

\subsection{Subjects and protocol}

Consecutive patients were considered eligible if they met the Berlin definition of ALI/ARDS [19]: 1) acute onset; 2) oxygenation index (pressure partial pressure of oxygen ( $\mathrm{PaO} 2) /$ fraction of inspiration oxygen (FiO2) $<200 \mathrm{mmHg}$ to $\leq 300 \mathrm{mmHg}(1 \mathrm{mmHg}=0.133 \mathrm{kPa}) ; 3)$ chest imaging showing patchy shadows in both lungs; and 4) pulmonary arterial entrapment pressure $\leq 18 \mathrm{mmHg}$ or no clinical evidence of increasing left atrial pressure.

\subsection{Exclusion criteria}

Exclusion criteria were 1) less than 18 years old; 2) rapid progression to ARDS; 3) acute left heart failure or cardiogenic pulmonary edema; 4) patients with related intestinal diseases, such as ulcerative colitis and irritable bowel syndrome; 5) patients who had taken probiotics, antibiotics or immunosuppressants within the previous 2 weeks; and 6) tumors, diabetes, liver or kidney dysfunction, connective tissue disease, or other inflammatory diseases that may affect the Th17/Treg cell ratio and intestinal flora imbalance. Finally, two patients who met the inclusion criteria were recruited into the study. Twenty-five age and sex-matched healthy volunteers with no history of chronic disease served as controls. All 
subjects provided written informed consent. The Ethics Committee of Zhejiang Hospital approved the study, which was conducted according to the 1975 Declaration of Helsinki (as revised in 1983).

\subsection{Blood collection and analysis}

Feces were collected from each subject in the morning and stored at $-70^{\circ} \mathrm{C}$ until used. Tubes with heparin or ethylenediamine tetraacetate acid (EDTA) were used to collect the peripheral blood, and serum was obtained after centrifugation and stored at $-70^{\circ} \mathrm{C}$ for subsequent analyses. Blood gas analysis and white blood cell counts, neutrophils and C-reactive protein (CRP) were immediately tested in the clinical chemistry laboratory of Zhejiang Hospital.

\subsection{ALI induction and intervention}

C57BL/6 mice (Shanghai Slake Experimental Animal Co., Ltd., Shanghai, China) were obtained and housed at $20^{\circ} \mathrm{C}-26^{\circ} \mathrm{C}$ and $40-70 \%$ humidity. The ALI model was established via intratracheal instillation of LPS; the control group was instilled with the same amount of normal saline. The mice were randomly divided into the ALI, control, low-dose rhubarb (50 mg/kg), high-dose rhubarb $(150 \mathrm{mg} / \mathrm{kg})$, trichostatin A (TSA, $1 \mathrm{mg} / \mathrm{kg}$ ), and valproic acid (VPA, $200 \mathrm{mg} / \mathrm{kg}$ ) groups. The treatments were administered once daily for 5 days; the ALI and control groups received the same amount of normal saline via gavage.

\subsection{Sample collection}

The mice were sacrificed by cervical dislocation. Peripheral blood, lung tissue, spleen tissue and fecal samples were collected for subsequent analyses.

\subsection{Histopathology}

Lung tissues were fixed with $4 \%$ paraformaldehyde for $24 \mathrm{~h}$, dehydrated, routinely processed, and embedded in paraffin. The tissues were sectioned at $5-\mu \mathrm{m}$ thick and stained with hematoxylin and eosin $(\mathrm{HE})$ to analyze the pathological damage to the lung tissues.

\subsection{Enzyme-linked immunosorbent assay (ELISA)}

The lung tissue was ground and diluted 5 times with phosphate-buffered saline, then the lung tissue and peripheral blood were centrifuged at $3000 \mathrm{rpm}$ for $15 \mathrm{~min}$ and stored at $-70^{\circ} \mathrm{C}$ for detection. ELISA kits (CUSABIO, Wuhan, China) were used per the manufacturer's instructions to quantify the levels of histone deacetylation (HDAC)6 and HDAC9 in the serum and lung tissue.

\subsection{Flow cytometry assay}

Lymphocytes were collected from the spleen and peripheral blood, then stained with FITC-conjugated anti-CD4 (ebioscience, 11-0042-82, USA) and PE-conjugated anti-FOXP3 (ebioscience, 12-5773-82), and the number of $\mathrm{CD}^{+}{ }^{+} \mathrm{FOXP3}^{+}$Treg cells was detected via flow cytometry assays. After staining the cells with FITC-conjugated anti-CD 4 (ebioscience, 11-0042-82) and PE-IL-17A (ebioscience, 12-7177-81), the number of $\mathrm{CD} 4^{+} \mathrm{IL}-17^{+} \mathrm{Th} 17$ cells was also detected via flow cytometry assays. Flow cytometry analysis 
was performed on a FACSCalibur flow cytometer (BD Biosciences, San Jose, CA, USA) equipped with CellQuest software (BD Biosciences).

\subsection{Fecal bacterial DNA extraction and 16S rDNA sequencing}

The QIAamp DNA Stool Mini kit (Qiagen, Hilden, Germany) was used to extract fecal bacterial DNA per the manufacturer's protocols. The V3-V4 hypervariable regions of the qualified bacterial 16S rDNA were amplified via PCR using the GeneAmp PCR System 9700 (ABI Co., USA). The primers (upstream primer: 5'CTACGGGNGGCWGCAG-3'; downstream primer: 5'-GACTACHVGGGTWTCTAAT-3') were synthesized by Sangon Biotech (Shanghai, China). PCR was performed in 5- $\mu$ l volumes containing 0.1 units of Taq polymerase (Qiagen, Hilden, Germany), $10 \mathrm{ng}$ of whole-genome-amplified genomic DNA, 2.5 pmol of each PCR primer, and $2.5 \mathrm{pmol}$ of dNTP. The processing cycle was as follows: predenaturation at $95^{\circ} \mathrm{C}$ for 5 min, denaturation at $95^{\circ} \mathrm{C}$ for $30 \mathrm{~s}$, renaturation at $58{ }^{\circ} \mathrm{C}$ for $15 \mathrm{~s}$, and extension at $72{ }^{\circ} \mathrm{C}$ for $1 \mathrm{~min}$. The entire process was repeated for 40 cycles, followed by a final extension step at $72{ }^{\circ} \mathrm{C}$ for 10 min. The QIAquick PCR purification kit (Qiagen, Hilden, Germany) was used to recover and purify the PCR products, which were sequenced by Illumina MiSeq PE300 (Illumina, San Diego, CA, USA).

\subsection{Bioinformatics analysis of the gut microbiota}

After sequence alignment analysis using Usearch software, all sequences were clustered into operational taxonomic units (OTUs) according to $97 \%$ similarity. Metabolic pathways were used to explore the relationship between the intestinal microflora and metabolism using PICRUSt analysis. The a-diversity indices were used to evaluate the gut microbial community diversity and abundance. Principal coordinate analysis based on Bray-Curtis distance and UniFrac analysis was performed to compare the global microbiota composition in each group. The relative species composition abundances at the phylum, class, order, family and genus levels among groups were demonstrated in barplots, taxon assignment trees and a heatmap. Differential taxonomic features among groups were obtained using linear discriminant analysis (LDA) effect size (LEfSe) to identify taxonomic features that differed among groups. The top 15 species were used to construct the Spearman correlation heatmap using the Corrplot package of R software.

\subsection{Statistical analysis}

Statistical analyses were performed in R24 and GraphPad Prism 5.0 (GraphPad Software Inc., La Jolla, CA, USA). Data are presented as the mean \pm SD. Differences within groups were analyzed using t-tests, variance analysis was used for continuous variables, and chi-square tests were used for categorical variables. Significance was defined as $p<0.05$.

\section{Results}

\subsection{Participants' basic clinical characteristics}


Table 1 shows the participants' basic clinical characteristics. Two patients with ALI and 25 healthy subjects were included in the study. The mean age of the patients with ALI was $65.5 \pm 14.85$ years; the mean age of the control group was $60.42 \pm 11.35$ years. The partial pressure of oxygen ( $\mathrm{PaO} 2)$, partial pressure of carbon dioxide (PaCO2), $\mathrm{PaO} 2 / \mathrm{FiO} 2$ and $\mathrm{CRP}$ of the ALI patients differed significantly from those of the control group (all $p<0.05$ ).

Table 1

The basic clinical characteristics of subjects

ALI $(n=2)$ Control $(n=25) p$ value
Age (year) $65.5 \pm 14.8560 .42 \pm 11.350 .142$
$\mathrm{PH} 7.45 \pm 0.067 .39 \pm 0.050 .093$
$\mathrm{PaO} 2(\mathrm{mmHg}) 55.00 \pm 2.5582 .30 \pm 5.81<0.01$
$\mathrm{PaCO} 2(\mathrm{mmHg}) 34.50 \pm 4.1239 .62 \pm 6.120 .046$
$\mathrm{PaO} 2 / \mathrm{FiO} 2261.91 \pm 12.13391 .92 \pm 22.90<0.01$
$\mathrm{WBC}\left(* 10^{9} / \mathrm{L}\right) 7.60 \pm 3.275 .95 \pm 2.140 .073$
$\mathrm{NE}(\%) 61.55 \pm 4.8855 .32 \pm 2.100 .164$
$\mathrm{CRP}(\mathrm{mg} / \mathrm{L}) 27.20 \pm 35.962 .53 \pm 1.12<0.01$

Abbreviation: $\mathrm{PaO} 2=$ pressure partial pressure of oxygen, $\mathrm{PaCO} 2=$ partial pressure of carbon dioxide, $\mathrm{FiO2}=$ fraction of inspiration oxygen, $\mathrm{WBC}=$ white blood cell, $\mathrm{NE}=$ neutrophil, $\mathrm{CRP}=\mathrm{C}$ reactive protein.

\subsection{Intestinal flora sequencing in the ALI patients}

More than 40,000 sequences were obtained via Illumina MiSeq PE300 sequencing with an integrity $\geq$ 86.13\% (Fig. 1A). After sequence alignment analysis using Usearch software to remove low-quality sequences, 692 OTUs were obtained with $97 \%$ similarity. The 100 richest representative OTU sequences were used to build the phylogenetic tree using Muscle and FastTree software (Fig. 1B).

A pie chart was constructed to analyze the species distribution differences in the gut microbiotas between the ALI patients and healthy controls. Firmicutes and Bacteroidetes were the most abundant, with a proportion of $90 \%$. Firmicutes was significantly reduced and Bacteroidetes was markedly elevated in patients with ALI. At the phylum level, the percentages of Firmicutes and Bacteroidetes were $49.39 \%$ and $38.07 \%$ in the ALI patients and $64.57 \%$ and $26.34 \%$ in the controls, respectively. At the class and order levels, the percentages of Firmicutes and Bacteroidetes were $41.81 \%$ and $38.06 \%$ in the ALI patients and $52.58 \%$ and $26.33 \%$ in the controls, respectively (Fig. 2A, 2B, 2C). Thus, ALI inhibited Firmicutes and promoted Bacteroidetes.

\subsection{Associations between signaling pathways and the intestinal microbiota in ALI patients}


The Kyoto Encyclopedia of Genes and Genomes (KEGG) biochemical pathways were established via PICRUSt analysis to evaluate the relationship between the gut microbiota and metabolism in patients with ALI. Three-layer analyses were performed according to the hierarchical relationship of the signaling pathway. The first layer demonstrated that gut microbiota alterations were related to metabolism in ALI patients (Fig. 3A). Further analysis revealed a correlation between the intestinal microbiota and amino acid metabolites (Fig. 3B). The third-layer analysis indicated that histone metabolites were altered in ALI patients (Fig. 3C). Thus, changes in the intestinal flora affected histone metabolism in patients with ALI.

\subsection{Animal model of ALI}

\subsubsection{LPS-induced ALI model}

To verify the clinical results, a mouse model of ALI was induced by instilling varying LPS concentrations. Pathological damage to the lungs was evaluated via HE staining. HE staining indicated that increasing LPS concentrations aggravated the degree of lung injury (Fig. 4A). The ALI model induced with $3 \mathrm{mg} / \mathrm{L}$ of LPS was used in subsequent experiments. Rhubarb treatment markedly attenuated the lung tissue damage (Fig. 4B).

\subsubsection{Effect of rhubarb on the gut microbiota}

DNA was extracted from the feces for $16 \mathrm{~S}$ rDNA sequencing. Diversity analysis (observed species index and the species accumulation curve) indicated sufficient gut microbial community diversity, and the abundances did not markedly differ between groups (Fig. 5A, 5B). A Venn diagram was used to analyze the similarities and differences in OTUs among groups. Twenty-one OTUs were common among the six groups, and each group had its own unique OTUs (Fig. 5C). $\beta$-diversity analysis showed that the control group species differed markedly from those of the other groups (Fig. 5D-5F). LPS-induced ALI reduced the Firmicutes abundance, including Lactobacillales and Clostridiales, and increased the Bacteroidetes abundance, including Anaeroplasmatales, indicating that the gut microbiota was altered after LPSinduced acute lung injury.

The top 20 positive OTUs were selected as targets. Barplots of the phylum, class, order, family and genus levels (Fig. 6A-6C), taxon assignments (Fig. 6D) and heatmaps (Fig. 6E) were used to demonstrate the relative abundances in the microbiota. The gut microbiome consisted largely of Firmicutes, Bacteroidetes, Proteobacteria, and Tenericutes, of which, Firmicutes and Bacteroidetes accounted for $85 \%$ of the total bacteria. At the class and order levels, Bacteroidia and Clostridia were prevalent, accounting for most of the microbiota. Bacteroides and Lactobacillus were the most abundant genera. Interestingly, the change in the Firmicutes/Bacteroidetes ratio in the animal models was consistent with the results of the clinical study. In addition, the low-concentration rhubarb treatment repressed Anaeroplasmatales and did not significantly affect the Lactobacillus or Clostridiales abundances. Increasing the rhubarb concentration effectively promoted Lactobacillus proliferation. These results indicated that rhubarb treatment attenuated the gut microbiota dysbiosis in mice with ALI. 
LEfSe analysis was used to screen the bacteria that differed among groups at the genus level. The top 15 bacteria were included in the relative analysis of Firmicutes and Bacteroidetes. Firmicutes included Alistipes, Roseburia, Acetobacter, Clostridium, Oscillibacter, Intestimonas, Butyricicoccus, and Lactobacillus. Bacteroidetes included Bacteroides and Parabacteroides (Fig. 7A-7E). Firmicutes and Bacteroidetes were negatively correlated at different levels and positively correlated within the same phylum. Treatment with low-concentration rhubarb promoted Alistipes growth, which contributed to the growth of sclerenchymal cells and inhibited Bacteroides growth in mouse. High-concentration rhubarb promoted Intestinimonas growth, which contributed to Clostridiales and Lactobacillus proliferation and inhibited Bacteroides proliferation (Fig. 7F), indicating that treatment with high-concentration rhubarb inhibited Bacteroides growth by promoting Clostridium growth in mice with ALI.

\subsubsection{Effect of rhubarb on HDAC and Th17/Treg ratios}

Peripheral blood and lung tissue were used to detect the effect of rhubarb on HDAC6 and HDAC9. Highconcentration rhubarb significantly increased the HDAC6 in both the lung tissue and serum (Fig. 8A, 8B) but did not affect the HDAC9 concentration (Fig. 8C, 8D), indicating that the elevated HDAC6 occurred after the rhubarb treatment. The Th17/Treg cell ratio was calculated to further evaluate the effect of rhubarb on HDAC6 acetylation function. Peripheral blood and spleen lymphocytes were collected to calculate the Treg cells via flow cytometry. The number of Treg cells in the peripheral lymphocytes was opposite that in spleen (Fig. 9A). These results indicated that numerous Treg cells were produced in the spleen to replenish the Treg cells consumed in the peripheral blood during ALI. Because of the lymphocyte shortage in the peripheral blood, the spleen was used to detect Treg cells in the next experiment. The number of Th17/Treg cells was calculated via flow cytometry after treatment (Fig. 9B). The Treg cells were significantly reduced ( $* \star p<0.01$; Fig. 9 C), and the Th17 cells were significantly increased after rhubarb treatment ( ${ }^{*} p<0.05 ;$ Fig. 9D). Thus, rhubarb restored Th17/Treg cell ratios by increasing HDAC6 levels and exerting anti-inflammatory effects.

\section{Discussion}

The present research demonstrated that LPS-induced ALI led to a disproportionate Firmicutes/Bacteroidetes ratio with increased Bacteroidetes and decreased Firmicutes in the gut microbiota. Rhubarb treatment in mice alleviated gut microbiota dysbiosis, promoted Clostridium and Lactobacillus proliferation, increased HDAC functioning, induced Th17 cells to differentiate and mature, and exerted anti-inflammatory functions.

The gut microbiota is not typically altered in healthy individuals but can be affected by various disorders. Scientists have explored the differences in structure and abundance of the intestinal microflora under healthy and disease conditions to identify the role of the gut microbiota during disease development. Dysbiosis in the gut microflora also occurs in many disorders [15-17]. ALI activated the systemic inflammatory response and secretion of inflammatory mediators, leading to intestinal mucosal barrier damage and intestinal bacterial translocation, which affected the intestinal flora diversity, distribution 
and composition. We investigated the microflora characteristics in fecal matter from humans and mouse using $16 \mathrm{~S}$ rDNA sequencing and found significant differences in the species and their distributions between ALI patients and controls. The results showed higher Bacteroidetes abundances in the ALI patients than in the control group. Compared with the control group mice, the model group mice had an increased abundance of Bacteroidetes and decreased Firmicutes richness. The Firmicutes/ Bacteroidetes ratio plays a key regulatory role in ALI; this regulation was positively correlated among similar phyla and negatively correlated among different phyla. These data were consistent with those of previous studies on intestinal microbiota alterations in animal models of ALI. Li et al. found decreased Firmicutes in LPSinduced ALI models [7]. Sze et al. demonstrated that instilling LPS in the lungs led to acute changes in the cecal bacterial microbiota [8]. SCFAs, gut microbiota-derived bacterial products, regulate the size and function of Treg cells, affect the Th17/Treg cell ratio, and maintain the balance between pro- and antiinflammatory factors [20-21] The phylum Firmicutes includes the genera Alistipes, Roseburia, Acetobacter, Clostridium, Oscillibacter, Intestinimonas, Butyricicoccus, and Lactobacillus, which are important sources of SCFAs. Decreased Firmicutes can reduce SCFA production and cause a Th17/Treg cell imbalance. Therefore, dysbiosis of the gut microbiota with decreased Firmicutes may induce inflammation during ALI.

Rhubarb treatment effectively repaired the intestinal mucosal barrier and increased the abundances of Bifidobacterium and Lactobacillus. Neyrinck et al. [22] found that rhubarb extract restored the intestinal microbial ecosystem during alcohol-induced hepatic injury. A purified anthraquinone-glycoside preparation from rhubarb ameliorated gut microbiota dysbiosis, with an increase in probiotic Lactobacillus and other SCFA-producing Firmicutes species, Consistent with previous reports, the present study demonstrated that rhubarb supplementation contributed to Firmicutes proliferation and increased Clostridiales and Lactobacillus, inhibited Bacteroidetes proliferation, and attenuated the gut microbiota dysbiosis.

TSA can inhibit HDAC and induce histone H3 acetylation of the FOXP3 gene promoter in Treg cells, which restores the Th17/Treg cell balance [23]. A published study revealed that inhibiting HDAC6 blocked NF-KB activation by inhibiting IKB phosphorylation after an LPS challenge and alleviated LPS-induced acute lung inflammation [24]. Conversely, several papers have drawn opposite conclusions. Menden et al. [25] indicated that HDAC6 inhibition augmented LPS-induced acute lung inflammation. The imbalance in peripheral circulating Th17 and Treg cell frequencies gradually increased from mild to severe in ARDS patients, and a positively correlated with disease severity (9). Zhang et al. [26] reported that the Th17/Treg cell ratio in bronchiolar lavage fluid was higher after ALI induced by smoke inhalation. The results of Zhang's study were inconsistent with our results; we found that the HDAC6 concentration was elevated in both the lung tissue and serum, and the Th17/Treg ratio was decreased in the spleen in LPSinduced ALI. Rhubarb treatment increased the HDAC6 levels in the lung tissue and serum, thus reducing the Treg cells, increasing the Th17 cells, and restoring the Th17/Treg cell balance in the spleen. Several points accounted for the discrepancies in these results. To counteract the inflammation, Treg cells were consumed in the peripheral blood, then reproduced in the spleen to replenish the consumed cells. 
Rhubarb treatment attenuated the intestinal microbiota disorder and elevated the HDAC6 levels, which induced Th17 cell production and restored the splenic Th17/Treg cell balance.

A limitation of this study was that only two ALI patients were included, which may have increased the chance of bias. Second, we did not provide probiotics against the gut microbiota dysbiosis. Third, no SCFAs were detected in the present study. Future research should further explore the function of metabolic products in the gut microbiota and the effect of probiotics on inflammation in LPS-induced ALI.

\section{Conclusions}

The current study showed that ALI with intestinal microflora dysbiosis presented reduced Firmicutes and elevated Bacteroidetes in both human patients and ALI-induced mice, leading to a Th17/Treg cell imbalance and aggravated inflammation. Rhubarb played an anti-inflammatory role by contributing to Firmicutes proliferation.

\section{Abbreviations}

ALI=Acute lung injury, ARDS=Acute respiratory distress syndrome, SCFA=Short-chain fatty acid, EDTA=Ethylenediamine tetraacetate acid, $\mathrm{CRP}=\mathrm{C}$-reactive protein, $\mathrm{TSA}=$ Trichostatin $\mathrm{A}, \mathrm{VPA}=\mathrm{Valproic}$ acid, $\mathrm{HE}=$ Hematoxylin and eosin, ELISA=Enzyme-linked immunosorbent assay, HDAC=Histone deacetylation, $\mathrm{PaO} 2=$ partial pressure of oxygen, $\mathrm{PaCO} 2=$ partial pressure of carbon dioxide, $\mathrm{FiO2}=$ fraction of inspiration oxygen, WBC=white blood cell, NE=neutrophil, KEGG=Kyoto Encyclopedia of Genes and Genomes.

\section{Declarations}

Ethics approval and consent to participate: The study was conducted according to the World Medical Association Declaration of Helsinki in 1975, as revised in 1983, and was approved by the Ethic Committee of Zhejiang Hospital. All subjects provided their informed written consent.

Consent for publication: Not applicable.

Availability of data and materials: The dataset of this article are stored in the respiratory department of Zhejiang Hospital and can be made available upon request by contacting corresponding author.

Competing interests: The authors declare that they have no competing interests.

Funding: Natural Science Foundation of Zhejiang Province (LY18H290008), Natural Science Foundation of China (81973590) and the Medical and Health Science and Technology Plan of Zhejiang Province (2019309530) provided financial support in the form of researcher funding. The sponsor had no role in the design or conduct of this research. 
Authors' contributions: TYT drafted the manuscript, WF and YW collected the samples and analyze data, TZ contributed to perform detection and prepare tables, LZJ conceived and planned the study design. All authors read and approved the final manuscript.

\section{Acknowledgments}

We thank Traci Raley, MS, ELS, from Liwen Bianji, Edanz Editing China (www.liwenbianji.cn/ac) for editing a draft of this manuscript.

\section{References}

1. Gotts JE, Matthay MA. Treating ARDS: new hope for a tough problem. Lancet Respir Med 2014; 2(2): 84-85

2. Calfee CS, Eisner MD, Ware LB, Thompson BT, Parsons PE, Wheeler AP, et al. Trauma-associated lung injury differs clinically and biologically from acute lung injury due to other clinical disorders. Crit Care Med 2007; 35: 2243-2250.

3. Rubenfeld GD, Caldwell E, Peabody E, Weaver J, Martin DP, Neff M, et al. Incidence and outcomes of acute lung injury. N Engl J Med 2005; 353: 1685-1693.

4. Sommer F, Anderson JM, Bharti R, Raes J, Rosenstiel P. The resilience of the intestinal microbiota influences health and disease. Nat Rev Microbiol 2017; 15: 630-638.

5. Zimmermann Petra, Messina Nicole, Mohn William W, Finlay B Brett, Curtis Nigel. Association between the intestinal microbiota and allergic sensitization, eczema, and asthma: A systematic review. J Allergy Clin Immunol 2019; 143: 467-485.

6. Souza DG, Vieira AT, Soares AC, Pinho V, Nicoli JR, Vieira LQ, et al. The essential role of the intestinal microbiota in facilitating acute inflammatory responses. J Immunol 2004; 173: 4137-4146.

7. Li Y, Liu XY, Ma MM, Qi ZJ, Zhang XQ, Li Z, et al. Changes in intestinal microflora in rats with acute respiratory distress syndrome. World J Gastroenterol 2014; 20: 5849-5858.

8. Sze MA, Tsuruta M, Yang SW, Oh Y, Man SF, Hogg JC, et al. Changes in the bacterial microbiota in gut, blood, and lungs following acute LPS instillation into mice lungs. PLoS One 2014; 9: e111228.

9. Yu ZX, Ji MS, Yan J, Cai Y, Liu J, Yang HF, et al. The ratio of Th17/Treg cells as a risk indicator in early acute respiratory distress syndrome. Crit Care 2015; 19: 82. 10. Luo A, Leach ST, Barres R, Hesson LB, Grimm MC, Simar D. The microbiota and epigenetic regulation of T helper 17/regulatory T cells: in search of a balanced immune system. Front Immunol 2017; 8: 417.

10. Luo S, Wen R, Wang Q, Zhao Z, Nong F, Fu Y. et al. Rhubarb Peony Decoction ameliorates ulcerative colitis in mice by regulating gut microbiota to restoring Th17/Treg balance. J Ethnopharmacol. 2019;231:39-49.

11. Huang $Z$, Xu Y, Wang Q, Gao X. Metabolism and mutual biotransformations of anthraquinones and anthrones in rhubarb by human intestinal flora using UPLC-Q-TOF/MS. J Chromatogr B Analyt Technol Biomed Life Sci. 2019;1104:59-66. 
12. Wang Z, Elekwachi C, Jiao J, Wang M, Tang S, Zhou C. et al. Changes in Metabolically Active Bacterial Community during Rumen Development, and Their Alteration by Rhubarb Root Powder Revealed by 16S rRNA Amplicon Sequencing. Front Microbiol. 2017;8:159.

13. Yao P, Cui M, Li Y, Deng Y, Wu H. Effects of rhubarb on intestinal flora and toll-like receptors of intestinal mucosa in rats with severe acute pancreatitis. Pancreas. 2015;44:799-804.

14. Xia S, Ni Y, Zhou Q, Liu H, Xiang H, Sui H. et al. Emodin attenuates severe acute pancreatitis via antioxidant and anti-inflammation activity. Inflammation. 2019;42:2129-38.

15. Song YD, Li XZ, Wu YX, Shen Y, Liu FF, Gao PP. et al. Emodin alleviates alternatively activated macrophage and asthmatic airway inflammation in a muring asthma model. Acta Pharmacol Sin. 2018;39:1317-25.

16. Xiao M, Zhu T, Zhang W, Wang T, Shen YC, Wan QF. et al. Emodin ameliorates LPS-induced acute lung injury, involving the inactivation of NF-KB in mice. Int J Mol Sci. 2014;15:19355-68.

17. Zeng YQ, Dai Z, Lu F, Lu Z, Liu X, Chen C. et al. Emodin via colonic irrigation modulates gut microbiota and reduces uremic toxins in rats with chronic kidney disease. Oncotarget. 2016;7:17468-78.

18. ARDS Definition Task Force. Ranieri VM, Rubenfeld GD, Thompson BT, Ferguson ND, Caldwell E, et al Acute respiratory distress syndrome: the Berlin Definition. JAMA 2012; 307: 2526-33.

19. Smith PM, Howitt MR, Panikov N, Michaud M, Gallini CA, Bohlooly-Y M, et al. The microbial metabolites, short-chain fatty acids, regulate colonic Treg cell homeostatsis. Science. 2013;341:56973.

20. Arpaia N, Campbell C, Fan X, Dikiy S, van der Veeken J, et al. Metabolites produced by commensal bacteria promote peripheral regulatory T-cell generation. Nature. 2013;504:451-5.

21. Neyrinck AM, Etxeberria U, Taminiau B, Daube G. Van Hul M, Everard A, et al. Rhubarb extract prevents hepatic inflammation induced by acute alcohol intake, an effec related to the modulation of the gut microbiota. Mol Nutr Food Res 2017; 61(1).

22. Tao R, de Zoeten EF, Ozkaynak E, Chen C, Wang L, Porrett PM, et al. Deacetylase inhibtion promotes the generation and function of regulatory T cells. Nat Med. 2007;13:1299-307.

23. Liu L, Zhou X, Shetty S, Hou G, Wang Q, Fu J. HDAC6 inhibition blocks inflammatory signaling and caspase-1 activation in LPS-induced acute lung injury. Toxicol Appl Pharmacol. 2019;370:178-83.

24. Menden H, Xia S, Mabry SM, Noel-MacDonnell J, Rajasingh J, Ye SQ. et al. Histone deacetylase 6 regulates endotheliao MyD88-dependent canonical TLR signaling, lung inflammation, and alveolar remodeling in the developing lung. Am J Physiol Lung Cell Mol Physiol. 2019;317:L332-46.

25. Zhang F, Li MY, Lan YT, Wang CB. Imbalance of Th17/Tregs in rats with smoke inhalation-induced acute lung injury. Sci Rep. 2016;6:21348.

\section{Figures}


A

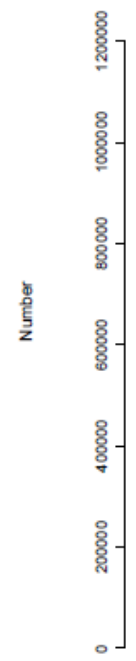

Sequence length distribution

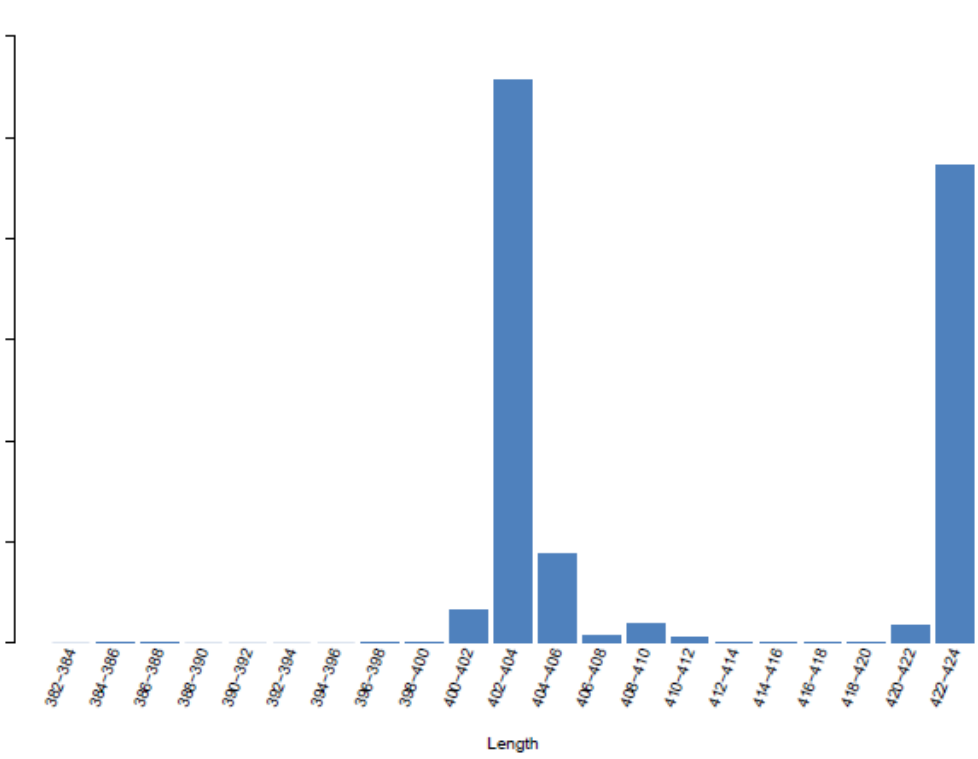

B

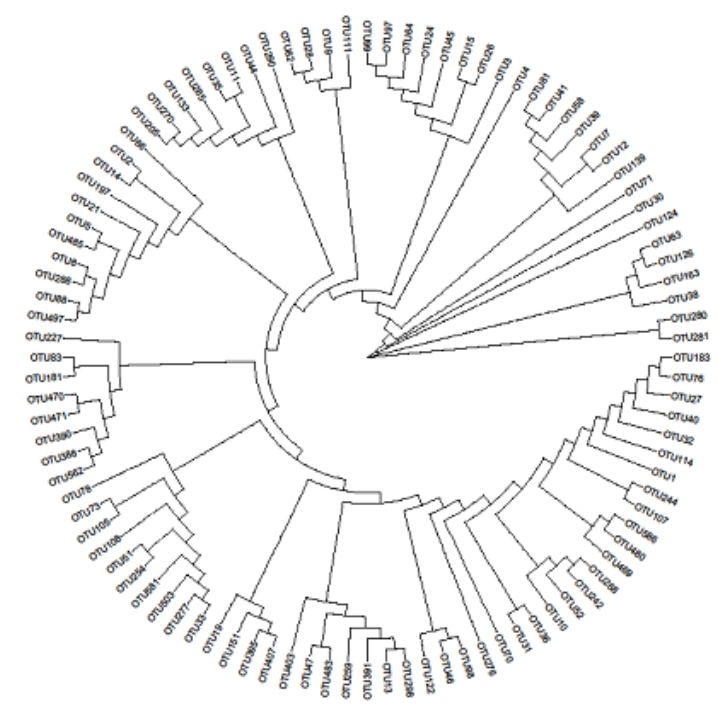

Figure 1

More than 40,000 sequences with $\geq 86.13 \%$ integrity were obtained via Illumina MiSeq PE300 sequencing (A). Phylogenetic tree showing evolutionary relationships among species (B). 

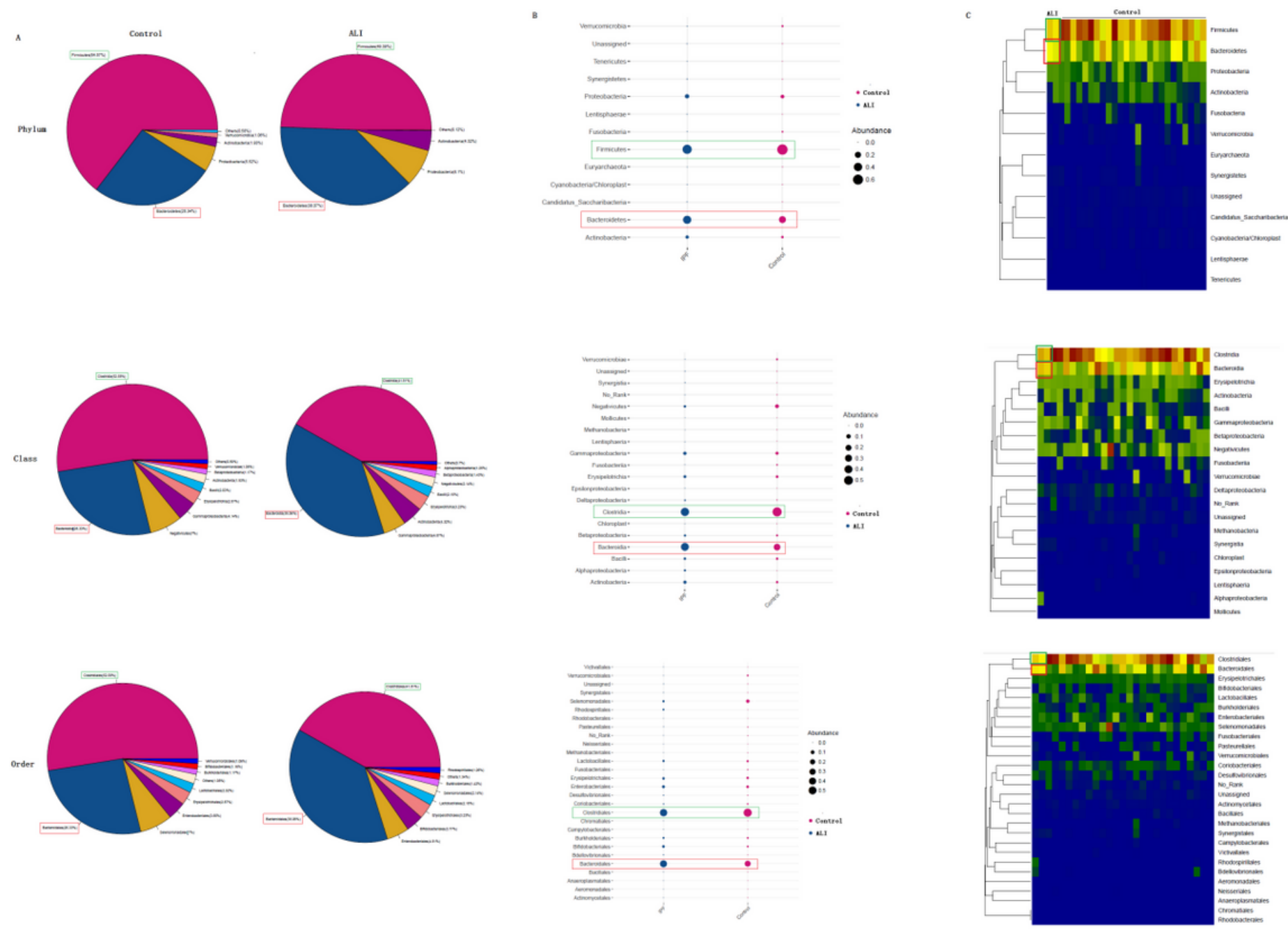

Figure 2

Percentages of Firmicutes/Bacteroidetes at the phylum, class and order levels in ALI patients and controls by pie charts $(A)$, bubble charts $(B)$, and heatmaps $(C)$. 
A
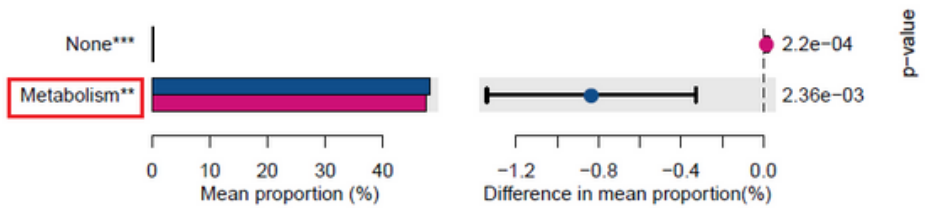

B

$\square$ Control $\square$ ALI

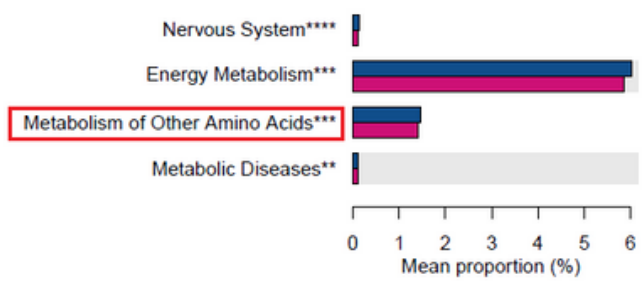

C

$\square$ Control $\square$ ALI

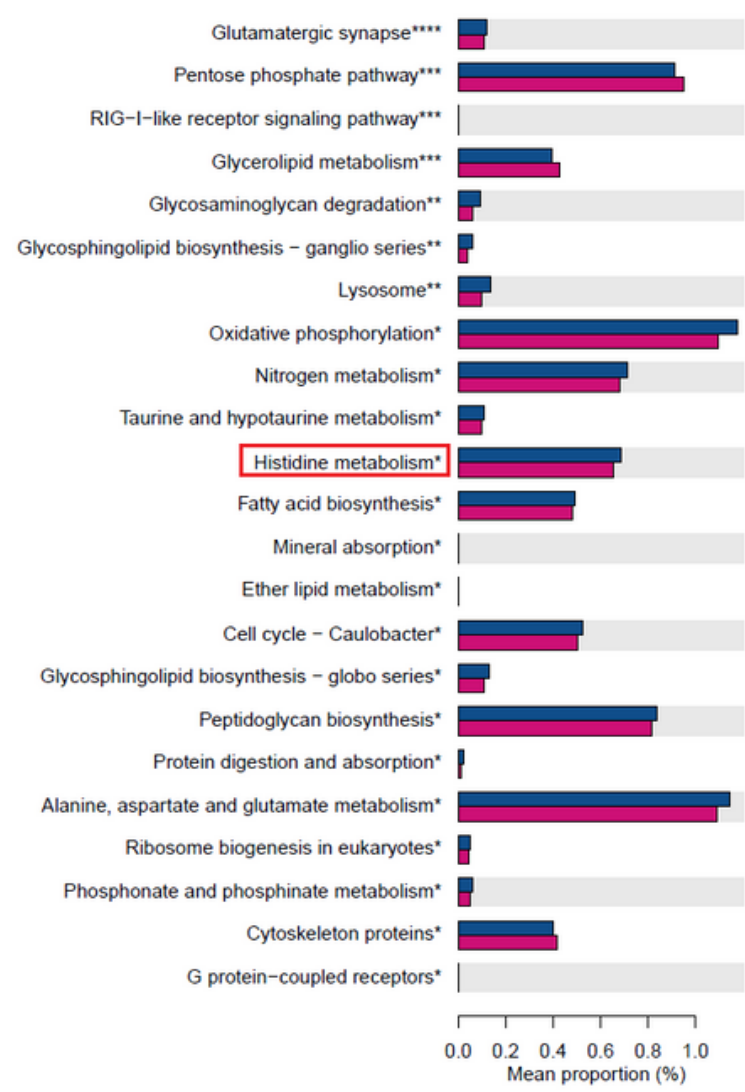

$95.0 \%$ confidence intervals

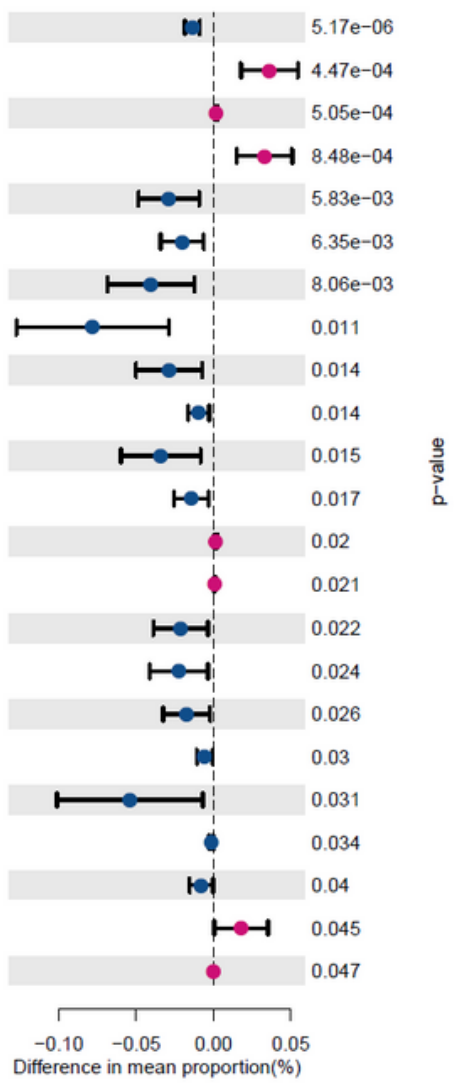

\section{Figure 3}

KEGG biochemical pathways established via PICRUSt. Association between the gut microbiota and metabolism in ALI patients (A). Correlation between the intestinal microbiota and amino acid metabolites (B). Gut microflora alterations associated with histone metabolites (C). 
A

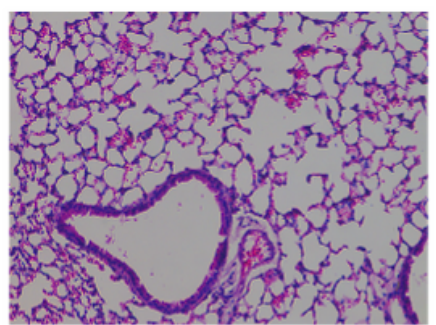

Control

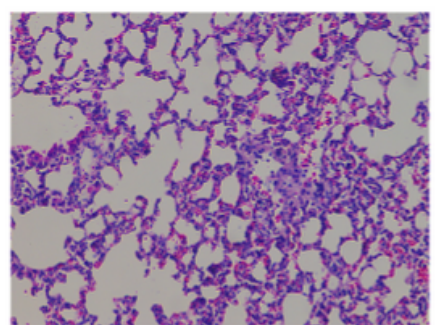

2mg LPS

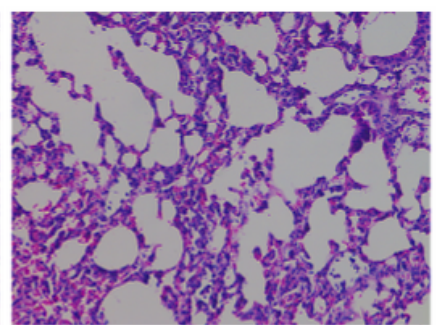

2.5mg LPS

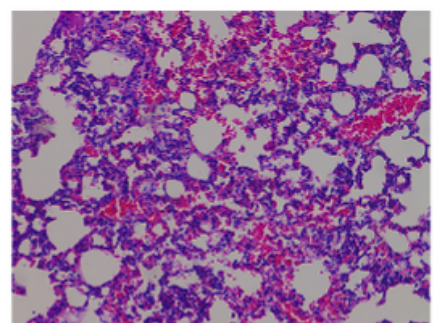

$3 \mathrm{mg}$ LPS

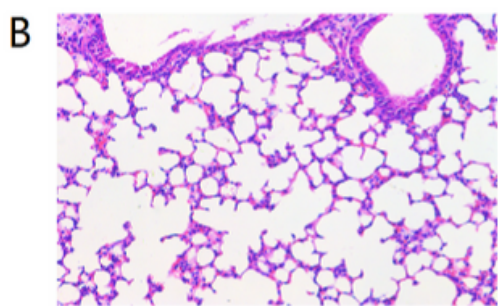

Control

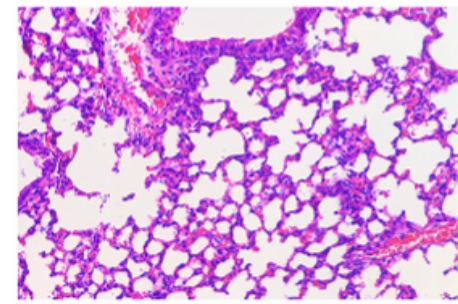

ALI treat with TSA

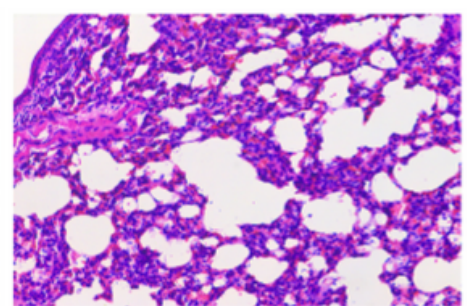

ALI model

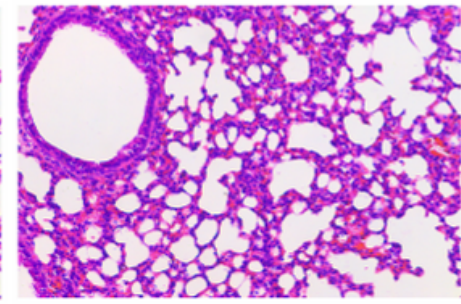

ALI treat with SCFA

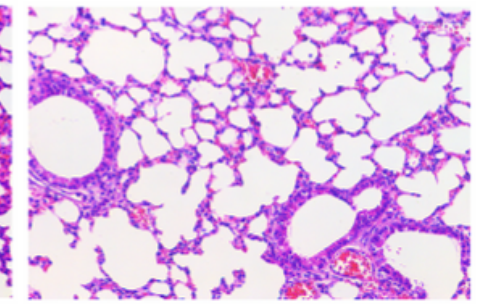

ALI treat with

$50 \mathrm{mg} / \mathrm{kg}$ rheum

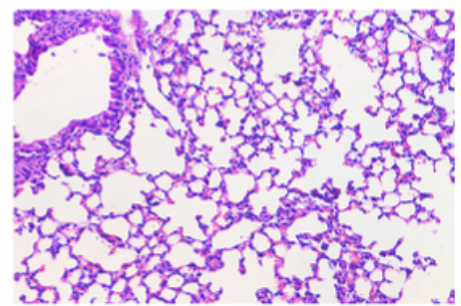

ALI treat with

$150 \mathrm{mg} / \mathrm{kg}$ rheum

Figure 4

ALI model was established by different concentration of LPS, the degree of lung injury aggravated with the increasing of LPS concentration (Fig 4A). After rhubarb or other intervention, the damage of lung tissue was remarkable improved (Fig 4B). 
A
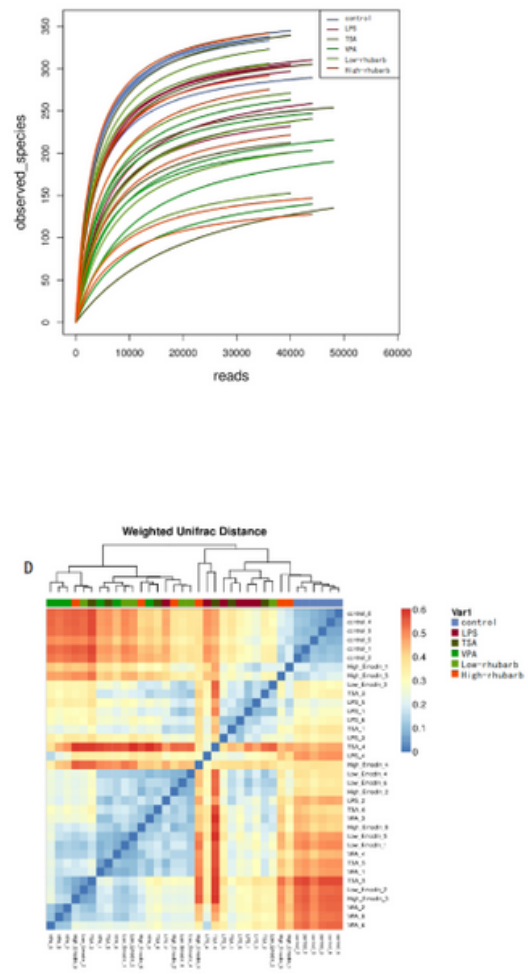

B

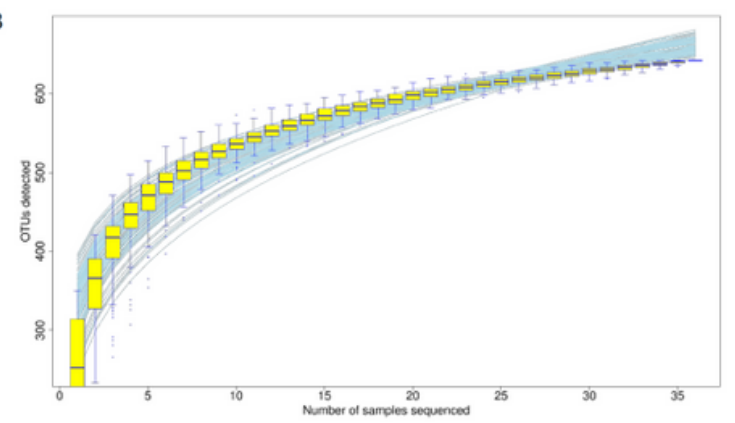

C
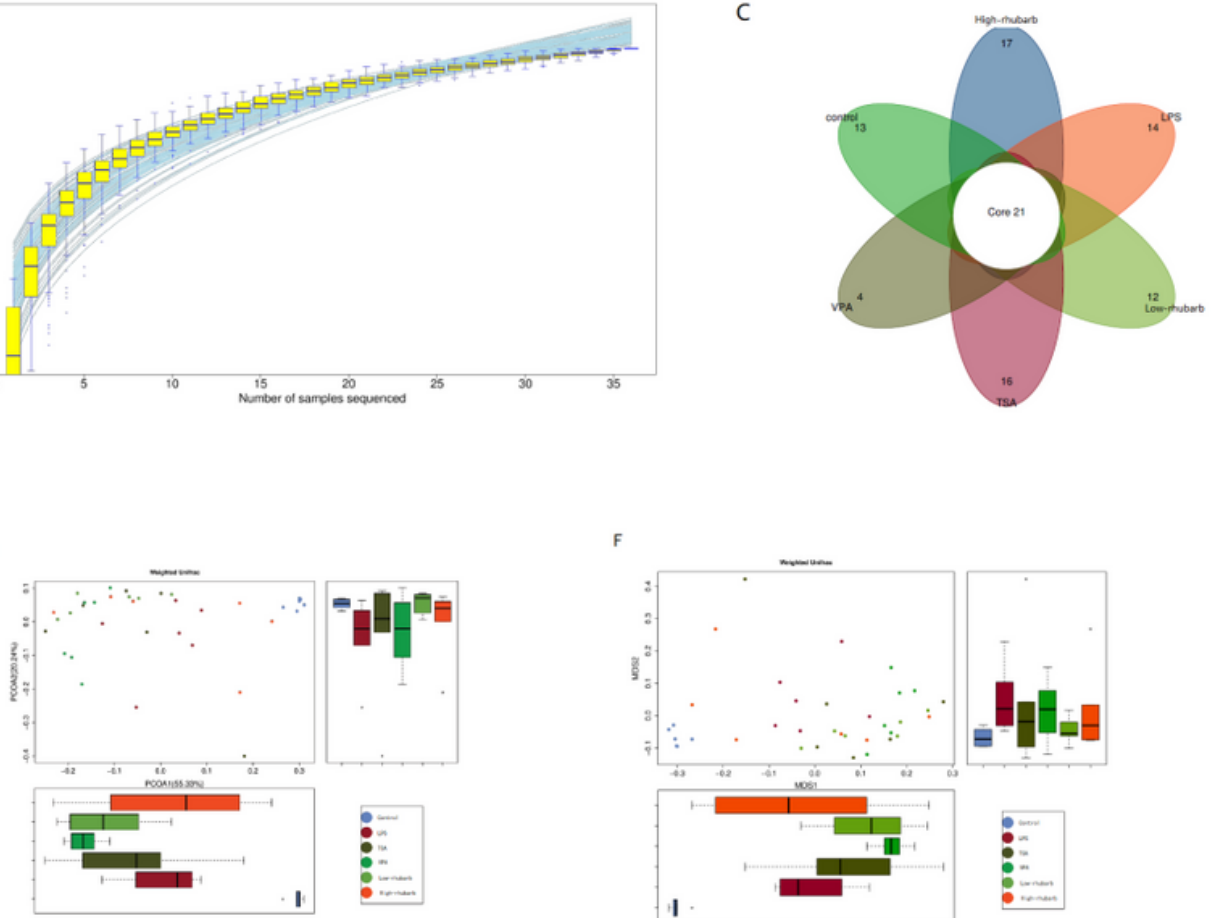

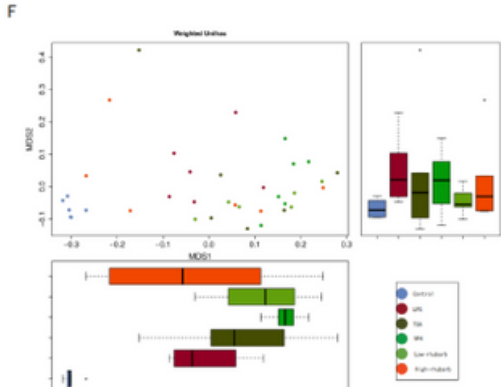

Figure 5

Diversity analysis indicated sufficient gut microbial community diversity and abundances via the observed species index (A) and the species accumulation curve (B). Venn diagram showing the OTU abundances per sample and the common and unique OTUs among groups (C). $\beta$-diversity analysis showing marked species differences between the control and intervention groups (D-F). 

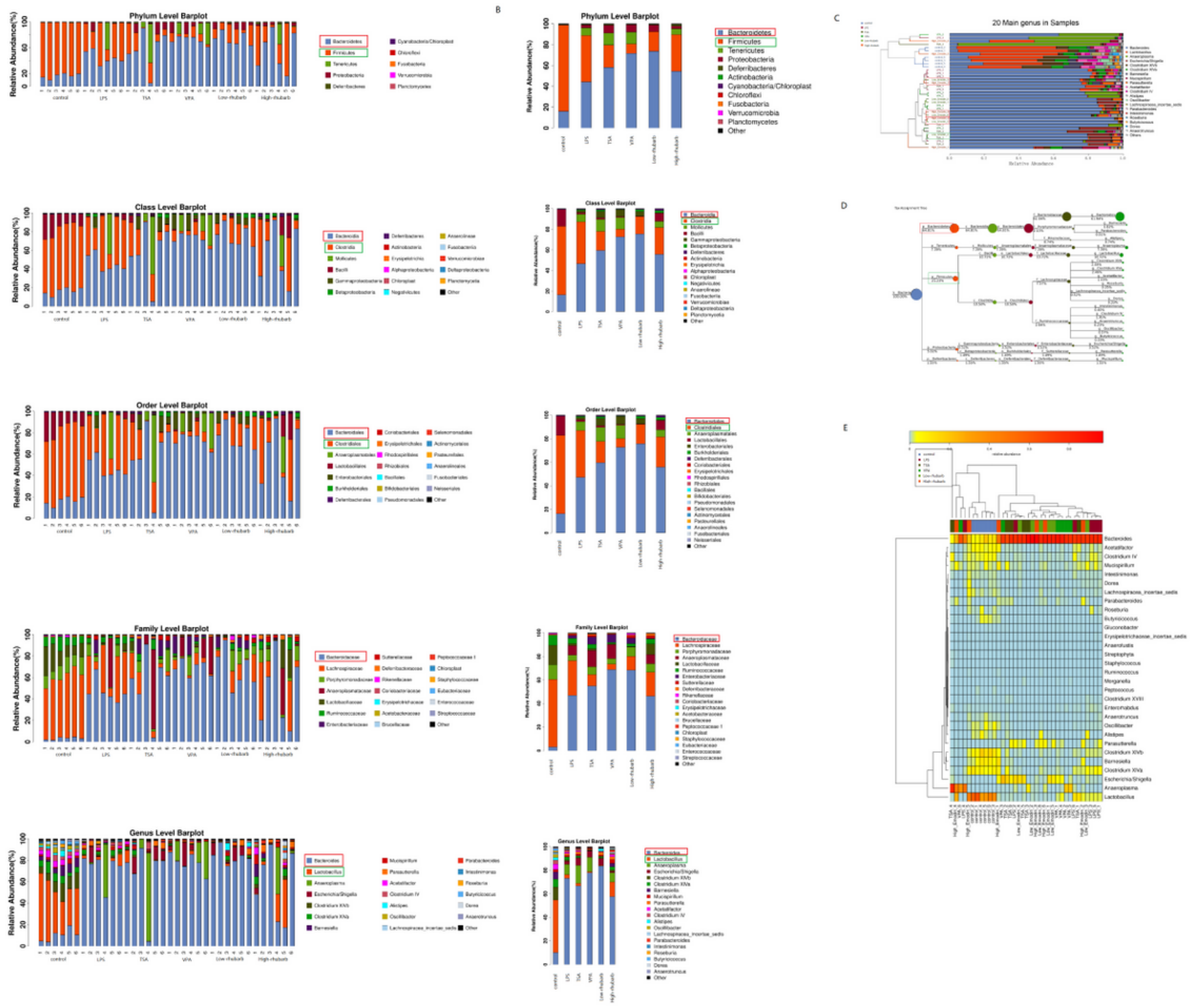

Figure 6

Barplots of the phylum, class, order, family and genus levels (A-B), species abundance cluster (C), taxon assignment tree (D) and heatmap (E) demonstrating the relative abundances of microorganisms at different levels among groups. 

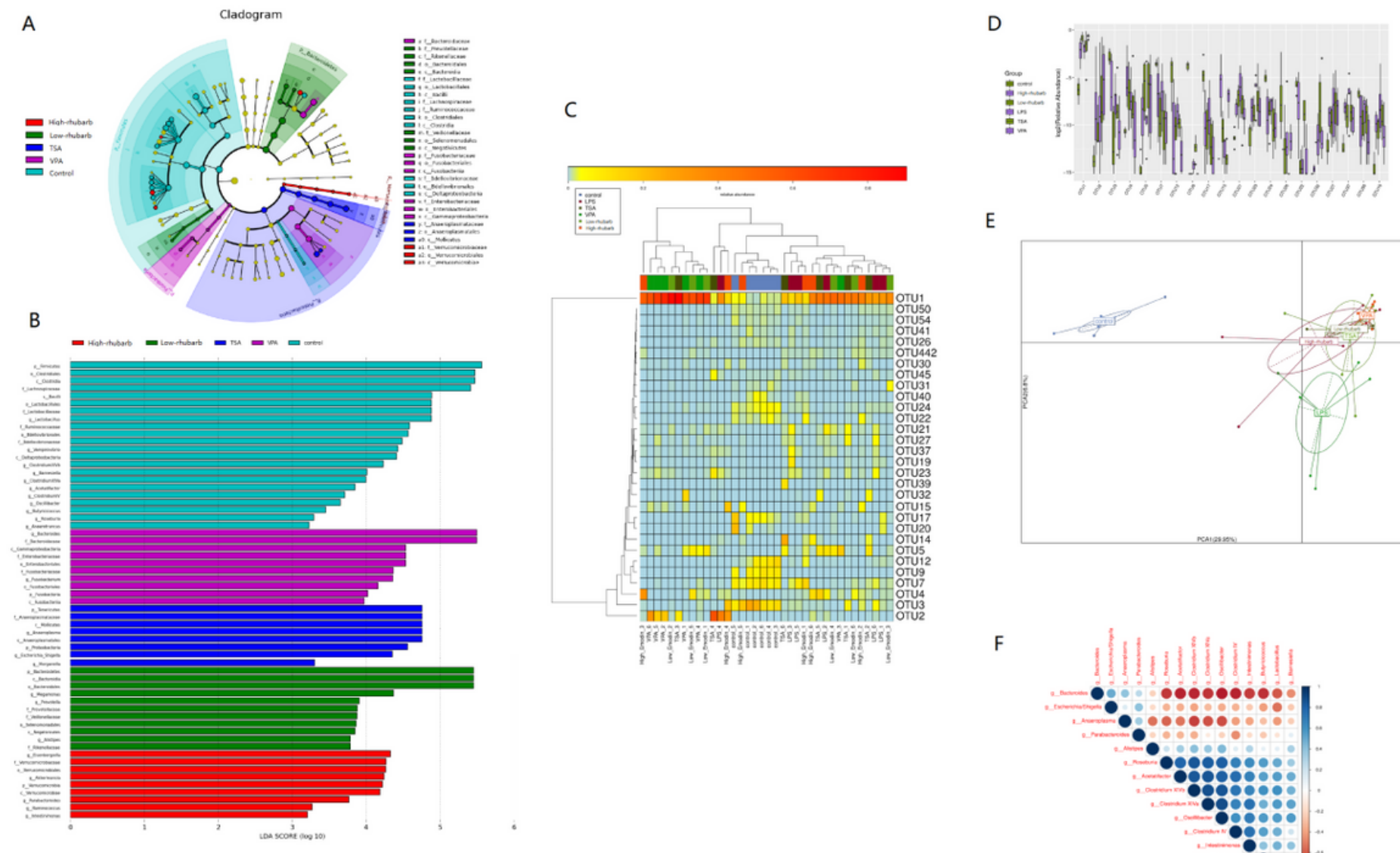

E
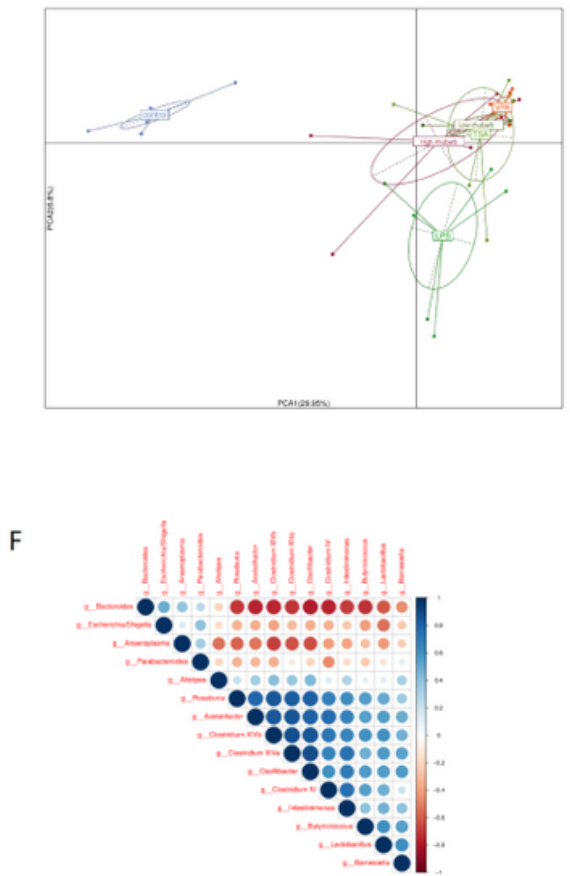

Figure 7

Cladogram used to screen the bacteria that differed markedly among groups (A). Histogram of LDA score distributions showing the LDA scores of the microbial groups with significant differences (B). Heatmap (C), box plot (D) and scatter plot (E) demonstrating significantly different species between groups. Top 15 species selected to analyze correlations at the genus level. Bacteroidetes (red frame) included Bacteroides and Parabacteroides; Firmicutes (green frame) contained Roseburia, Acetobacter, Clostridium, Oscillibacter, Intestimonas, Butyricicoccus and Lactobacillus (F). 
A

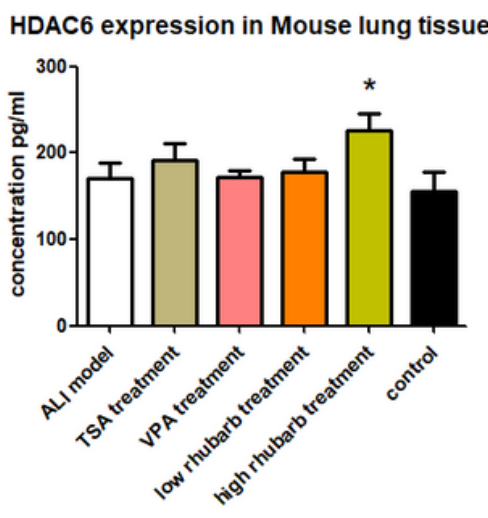

D

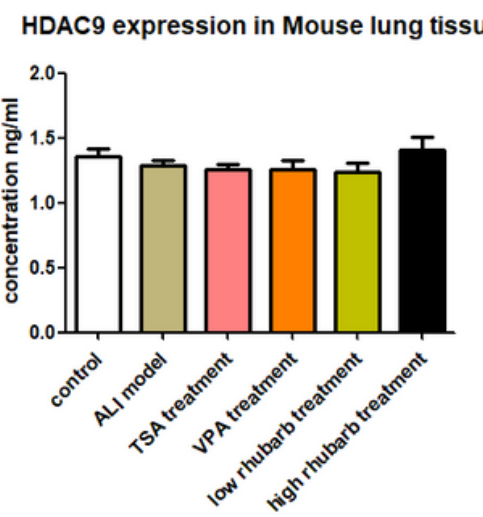

B

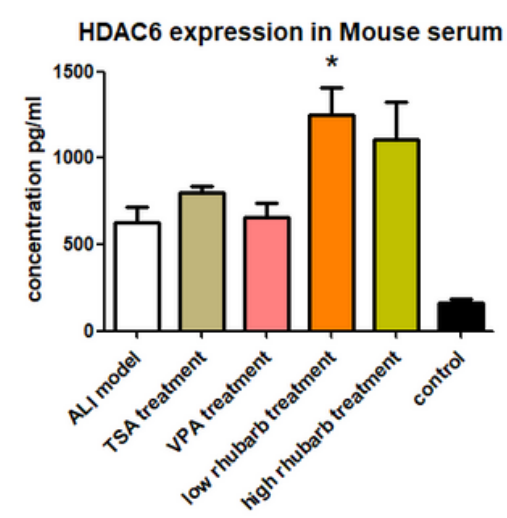

E

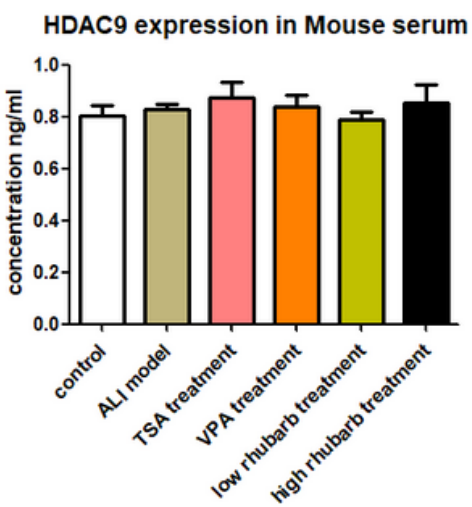

C

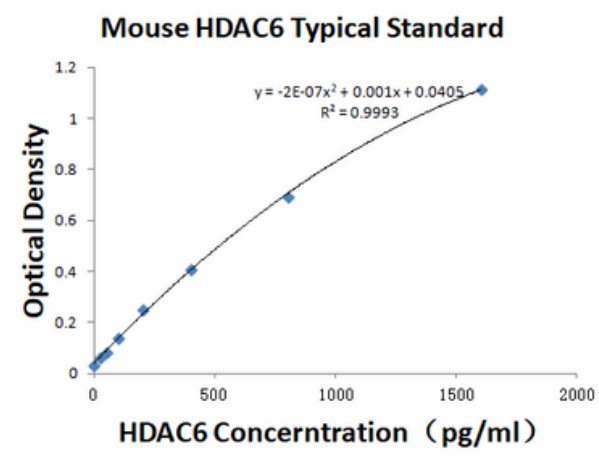

$\mathrm{F}$

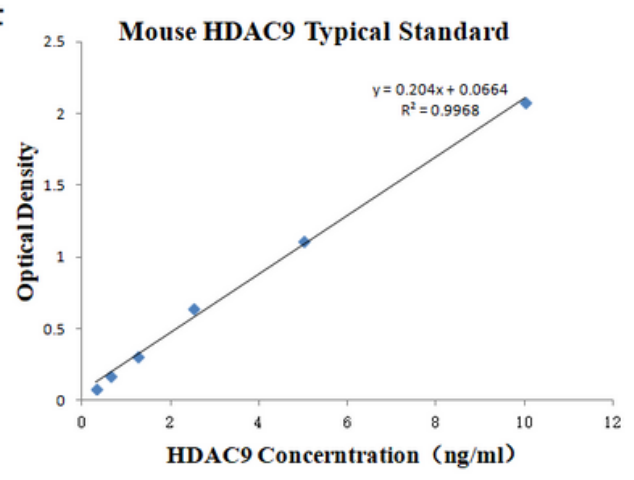

Figure 8

After high dose rhubarb treatment, the level of HDAC6 was significantly higher than other groups from lung tissue $\left({ }^{\star} p<0.05\right)(\mathrm{Fig} 8 \mathrm{~A})$. Compared with control group, the level of HDAC6 in all groups was higher in the peripheral blood serum ( ${ }^{\star} \mathrm{p}<0.05$ ) (Fig 8B). HDAC6 test standard curve (Fig 8C). Whether peripheral blood or lung tissue, no change was identified in HDAC9 activity (Fig 8D, 8E). HDAC9 test standard curve (Fig 8F). 
A
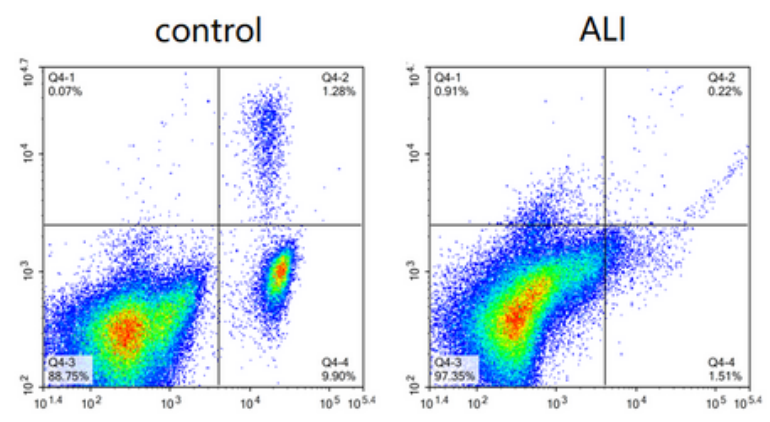

whole blood

뜬
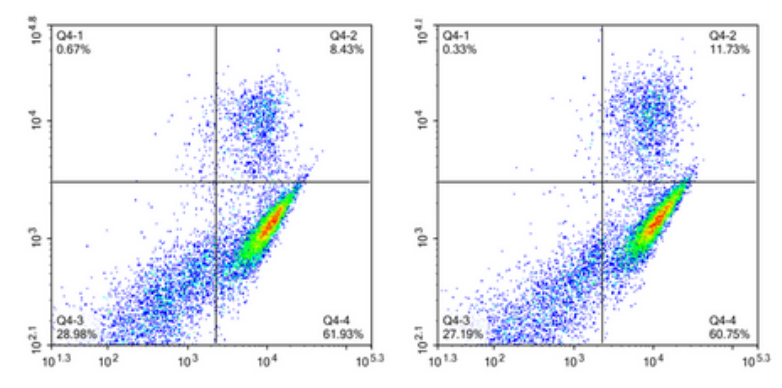

spleen

FITC

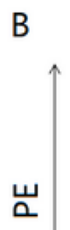
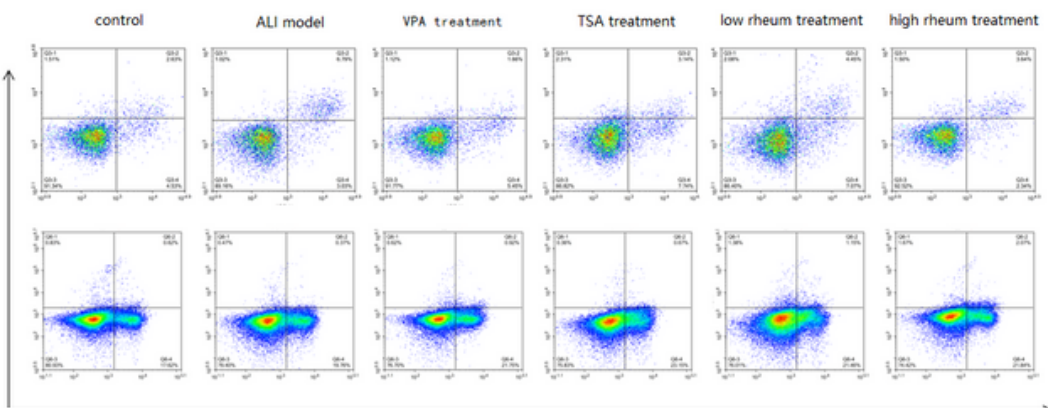

Treg
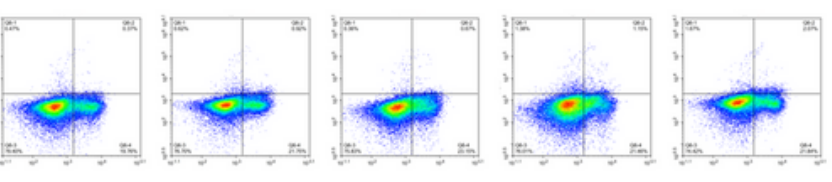

Th17

FITC

C

The percentage of Treg expression Mouse spleen

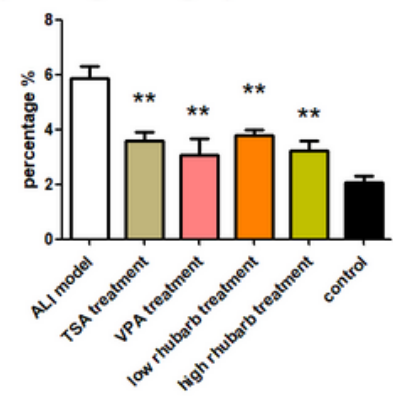

D The percentage of Th17 expression Mouse spleen

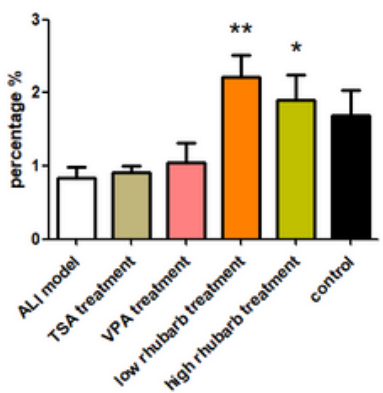

\section{Figure 9}

Flow cytometry was used to detect the percentages of Treg cells in the peripheral blood and spleen tissues. Treg cells were decreased in the peripheral blood and increased in the spleen tissue. Because few Treg cells were concentrated in the peripheral blood, the spleen tissue was used to detect the Treg/Th17 percentage in subsequent experiments (A). Th17 and Treg cell numbers were determined via flow 
cytometry from spleen tissue (B). Treg cells were significantly reduced (C) (**p<0.01), and Th17 cells were significantly increased after rhubarb treatments (D) $\left({ }^{*} p<0.05\right)$. 